\title{
Les espèces ligneuses locales à huile : une ressource utile pour les communautés locales au Sud du Mali
}

\author{
Amadou Malé KOUYATE ${ }^{1 *}$, Urbain DEMBELE ${ }^{1}$ et Anne Mette LYKKE ${ }^{2}$ \\ ${ }^{1}$ Institut d'Economie Rurale, BP. 16, Sikasso, Mali. \\ ${ }^{2}$ Department of Bioscience, Aarhus University, Vejlsфvej 25, 8600, Silkeborg, Denmark. \\ *Auteur correspondant; E-mail: Kouyate01@hotmail.com; Tél : +2237516 52 19; \\ Fax : +22321620349
}

\section{REMERCIEMENTS}

Les auteurs tiennent à remercier DANIDA pour le financement du projet de recherche basée sur les arbres à huile en Afrique de l'Ouest (QualiTree, DFC10-002AU) qui a permis la réalisation du présent travail.

\section{RESUME}

La majorité des espèces ligneuses des galeries forestières restent sous-utilisées au Mali. Le present article cherche à connaître les usages des espèces à huile en fonction des groupes ethniques, des villages, de l'âge et du sexe. Le diagnostic a été mené auprès d'un échantillon de 96 personnes appartenant à 4 villages au Sud du Mali à partir d'interview semi-structurée. Les résultats ont indiqué une diversité d'utilisations de l'huile des espèces forestières locales étudiées selon les groupes ethniques, les villages, l'âge et le sexe. Leurs principales utilisations se résument à l'alimentation humaine, au traitement des maladies et des insectes et aux soins cosmétiques. Cette diversification d'utilisations peut constituer une raison pour entreprendre des actions de multiplication et de domestication de ces espèces qui sont en majorité sous-utilisées.

(C) 2015 International Formulae Group. All rights reserved.

Mots clés : Alimentation, cosmétique, ethnobotanique, médecine, galerie forestière.

\section{The native oil tree species: a useful resource for local communities in southern Mali}

\begin{abstract}
The majority of woody species of the forest gallery remain underutilized in Mali. This article seeks to understand the uses of oil trees species according to the ethnic group, village, age and gender. Semi-structured interviews were conducted among 96 people from 4 villages in southern Mali. The results showed several uses of oils from the studied native species, which differed according to ethnic group, village, age and gender. The main uses were food, medicine and insecticide, and cosmetics. This diversification of uses can constitute a reason to undertake propagation and domestication activities of these species which are mostly underutilized. (c) 2015 International Formulae Group. All rights reserved.
\end{abstract}

Keywords: Food, cosmetics, ethnobotany, medicine, forest gallery. 


\section{INTRODUCTION}

Les populations rurales des pays sahéliens dépendent fortement des formations forestières naturelles. Ces formations forestières contribuent à la conservation de la biodiversité, à l'amélioration des revenus et à la satisfaction des besoins de santé et d'alimentation des communautés rurales (Andel, 2006; Krohmer et al., 2006; FAO, 2007; Dawson et al., 2009; FAO, 2009). En plus du bois, les populations rurales ont des besoins tournés vers les produits forestiers non ligneux (PFNL). Ces PFNL sont constitués de biens d'origine biologique autres que le bois, provenant des forêts, des terrains boisés ou d'arbres hors forêts (Wong et al., 2001).

$\mathrm{Au}$ cours de ces dernières décennies, les PFNL ont été considérés comme un potentiel viable pour promouvoir simultanément le développement économique rural et la conservation des forêts (Dovie, 2003; Mahapatra et al., 2005; Biloso et Lejoly, 2006; Weber et al., 2010; Albert et Emmanuel, 2011; Schumann et al., 2011). Ces PFNL font également l'objet d'un intérêt croissant (Bikoué et Essomba, 2007). Au nombre des PFNL récoltés, les fruits sont très prisés des populations pour être mangés ou produire des huiles à partir des graines. Ces huiles ont toujours constitué une source de produits à usages alimentaire, cosmétique, médicinal et insecticide (Dronne et Forslund, 2009; Dembélé et al., 2015). Au regard de la démographie galopante et de l'élévation du niveau de vie des populations humaines, les besoins en huiles végétales deviennent de plus en plus croissants. Or, ces populations vivant en milieu rural ont une connaissance approfondie des produits forestiers non ligneux qui les environnent et des usages des plantes alimentaires et médicinales (Tchatat et Ndoye, 2006). Cependant, ces savoirs traditionnels sur les espèces d'arbres oléagineux locaux sont peu documentés. Aujourd'hui, ces savoirs sont de plus en plus menacés par les changements de l'environnement et du style de vie (Gruère et al., 2006; Krohmer et al., 2006). C'est pourquoi, il est intéressant de les intégrer dans les activités de sélection, de domestication, de sylviculture, de transformation et de valorisation (Gaoué et Ticktin, 2009) afin d'éviter leur déperdition. Cette quantification ethnobotanique englobe les aspects relatifs à l'analyse de la connaissance des populations sur les utilisations d'espèces de plantes (Medeiros et al., 2011).

Le présent article a été initié, à travers des enquêtes ethnobotaniques quantitatives dans les villages le long des galeries forestières au Sud du Mali. Il cherche à identifier les espèces d'arbres locaux à huile intéressantes pour l'alimentation humaine, la santé, la cosmétique et dans le traitement insecticide; livrer et estimer l'importance et les différences d'usages entre les groupe ethnique, les villages, l'âge et le sexe.

\section{MATERIEL ET METHODES \\ Zone d'étude}

La région de Sikasso au Mali est située entre 12 $30^{\prime}$ latitude Nord et la frontière ivoirienne d'une part et $8^{\circ} 45^{\prime}$ ' longitude Ouest et la frontière Burkinabé d'autre part. Elle couvre une superficie de $71790 \mathrm{~km}^{2}$ soit 5,8\% $\mathrm{du}$ territoire national avec une population estimée à 3,04 millions d'habitants en 2013 (DRPSIAP ,2014). Les précipitations moyennes annuelles varient de $500 \mathrm{~mm}$ (zone soudanienne) à plus de $1100 \mathrm{~mm}$ (zone guinéenne).

Les enquêtes ethnobotaniques ont été conduites dans les villages de Konkolikoro, Mandela, Niankorobougou et Ziékorodougou qui sont situés dans le cercle de Sikasso au Sud du Mali (Figure 1). Le cercle de Sikasso couvre une superficie de $15375 \mathrm{~km}^{2}$. Le climat est de type soudanien. La température annuelle moyenne est de $24{ }^{\circ} \mathrm{C}$ en période fraîche et de $27{ }^{\circ} \mathrm{C}$ en période chaude. La population est composée en majorité de 
Senoufos qui cohabitent avec d'autres ethnies comme les Samogos, les Peuls et les Bambaras (PROMISAM, 2006).

\section{Méthodes}

Les enquêtes ethnobotaniques quantitatives sur les arbres locaux à huile ont été menées suivant les méthodes décrites par certains auteurs (Lykke et al., 2004 ; Dossou et al., 2012). Ces méthodes reposent sur la description des différents usages des espèces ligneuses par les familles paysannes. Ces enquêtes ont été réalisées dans quatre villages pendant la saison sèche en 2011. Les enquêtes ont été faites par une équipe composée d'un sociologue, d'un agroéconomiste et d'un aménagiste forestier. Elles ont été réalisées auprès des groupes ethniques Bambara, Peul, Samogo et Sénoufo (Tableau 1), à l'aide de questionnaires semi-structurés et focalisés sur les usages des huiles. Dans chaque village, un échantillon de 6 femmes et 6 hommes par groupe ethnique a été interviewé individuellement, soit un total de 48 femmes et 48 hommes. Le choix de ces personnes par l'ensemble des villageois a été basé sur leurs connaissances avérées des plantes. Pour tenir compte de l'âge, les personnes interviewées ont été catégorisées comme suit : jeune (18 à 42 ans), adulte (42 à 82 ans) et vieille personne (plus de 82 ans).

\section{Traitement des données}

Pour chaque espèce, le pourcentage de citation a été calculé en divisant le nombre de répondants par le nombre total de personnes interrogées.

\section{Analyse statistique}

Les différences significatives entre les groupes ethniques, les villages, l'âge et le sexe ont été évaluées pour les différentes utilisations des espèces ligneuses qui ont été recensées à travers l'analyse de variance (Dagnélie 1977; Philippeau, 1986; Johnson et Wichern, 1998).

\section{RESULTATS \\ Diversité des espèces ligneuses à huile exploitées}

$\mathrm{Au}$ total, douze espèces ligneuses locales à huile appartenant à 7 familles ont été identifiées comme utilitaire dans la zone d'étude. Ce sont: Blighia sapida (Sapindaceae), Borassus aethiopum (Arecaceae), Carapa procera (Meliaceae), Elaeis guineensis (Arecaceae), Khaya senegalensis (Meliaceae), Lannea microcarpa (Anacardiaceae), Lophira lanceolata (Ochnaceae), Pentadesma butyracea (Clusiaceae), Vitellaria paradoxa (Sapotaceae).

\section{Les espèces ligneuses à huile pour l'alimentation humaine}

$\mathrm{Au}$ total, quatre espèces ont été identifiées pour l'alimentation alimentaire. Il s'agit de: Elaeis guineensis, Lophira lanceolata, Pentadesma butyracea et Vitellaria paradoxa. L'analyse de variance a permis de mettre en évidence de différences significatives entre les groupes ethniques, entre les villages, entre les jeunes, les adultes et les vieilles personnes, et entre les hommes et les femmes (Tableau 2). L'étude a montré une forte citation des espèces par les Sénoufos et les Samogos (100\%), de Vitellaria paradoxa dans le village de Mandela, d'Elaeis guineensis, de Lophira lanceolata et de Pentadesma butyracea dans le village de Niankorodougou (75 à 100\%), de Lophira lanceolata par les vieilles personnes (100\%), de Pentadesma butyracea par les femmes $(100 \%)$ et de Vitellaria paradoxa pour les hommes $(100 \%)$.

\section{Les espèces ligneuses à huile pour la fabrication du savon}

$\mathrm{Au}$ total, cinq espèces ont été identifiées pour la fabrication du savon. Ce sont Carapa procera, Khaya senegalensis, Lophira lanceolata, Pentadesma butyracea et Vitellaria paradoxa. L'analyse de variance a permis de montrer des différences 
significatives entre les groupes ethniques, entre les villages, entre les jeunes, les adultes et les vieilles personnes, et entre les femmes et les hommes pour l'utilisation de ces espèces (Tableau 2). L'étude a montré qu'une forte citation de Carapa procera et de Khaya senegalensis par les Sénoufos, de Lophira lanceolata et Pentadesma butyracea par les Samogos, de Carapa procera, de Pentadesma butyracea et de Khaya senegalensis par le village de Ziékorodougou, de Lophira lanceolata par le village de Niankorobougou et par les vieilles femmes (80 à 100\%).

Les espèces ligneuses à huile pour les soins de la peau

$\mathrm{Au}$ total, quatre espèces ont été identifiées pour les soins de la peau. Ce sont Carapa procera, Lophira lanceolata, Pentadesma butyracea et Vitellaria paradoxa. L'analyse de variance a permis de montrer des différences significatives entre les groupes ethniques, entre les villages, entre les jeunes, les adultes et les vieilles personnes, et entre les femmes et les hommes (Tableau 2). L'étude a montré une forte citation de Carapa procera et de Vitellaria paradoxa par les Sénoufos, de Carapa procera, de Lophira lanceolata et de Pentadesma butyracea par les Samogos, de Carapa procera et de Vitellaria paradoxa (83\%) à Ziékorodougou, de Carapa procera $(62 \%)$ à Niankorobougou et par les vieilles personnes (67\%), et de Pentadesma butyracea et de Vitellaria paradoxa par les femmes.

Les espèces ligneuses à huile pour les soins des cheveux

$\mathrm{Au}$ total, trois espèces ont été identifiées pour les soins des cheveux. Ce sont Lophira lanceolata, Pentadesma butyracea et Vitellaria paradoxa. L'analyse de variance a permis de mettre en évidence des différences significatives entre les groupes ethniques, entre les villages, entre les jeunes, les adultes et les vieilles personnes, entre les femmes et les hommes (Tableau 2). L'étude a montré une forte citation de Pentadesma butyracea par les Samogos $(80 \%)$ et les Bambaras (52\%), de Lophira lanceolata dans le village de Niankorobougou (75\%), et de Pentadesma butyracea, de Lophira lanceolata et de Vitellaria paradoxa par les femmes (70\%).

Les espèces ligneuses à huile pour la médecine traditionnelle

$\mathrm{Au}$ total, quatre espèces ont été identifiées pour les soins médicinaux. Ce sont Carapa procera, Elaeis guineensis, Khaya senegalensis et Vitellaria paradoxa. L'analyse de variance a permis de montrer des différences significatives entre les groupes ethniques, entre les villages, entre les jeunes, les adultes et les vieilles personnes, entre les femmes et les hommes (Tableau 2). L'étude a montré la citation faible de Khaya senegalensis (2\%) par les Peuls, une forte citation de Carapa procera par le village de Niankorobougou (90\%), de Carapa procera et d'Elaeis guineensis par les vieilles personnes (50-67\%) et de Vitellaria paradoxa par les femmes $(68 \%)$.

\section{Les espèces ligneuses à huile comme insecticide}

$\mathrm{Au}$ total, sept espèces ont été identifiées comme insecticide. Ce sont Borassus aethiopum, Khaya senegalensis, Lannea microcarpa, Raphia sudanica, Sterculia setigera, Vitellaria paradoxa et Ximenia americana. L'analyse de variance a permis de montrer des différences significatives entre les groupes ethniques et entre les villages (Tableau 2). L'étude a montré une forte citation de Borassus aethiopum, de Khaya senegalensis, de Lannea microcarpa, de Raphia sudanica, de Sterculia setigera et de Ximenia americana par les Samogos et les Bambaras (100\%), de Khaya senegalensis, de Vitellaria paradoxa et de Sterculia setigera par le village de Ziékorodougou. 


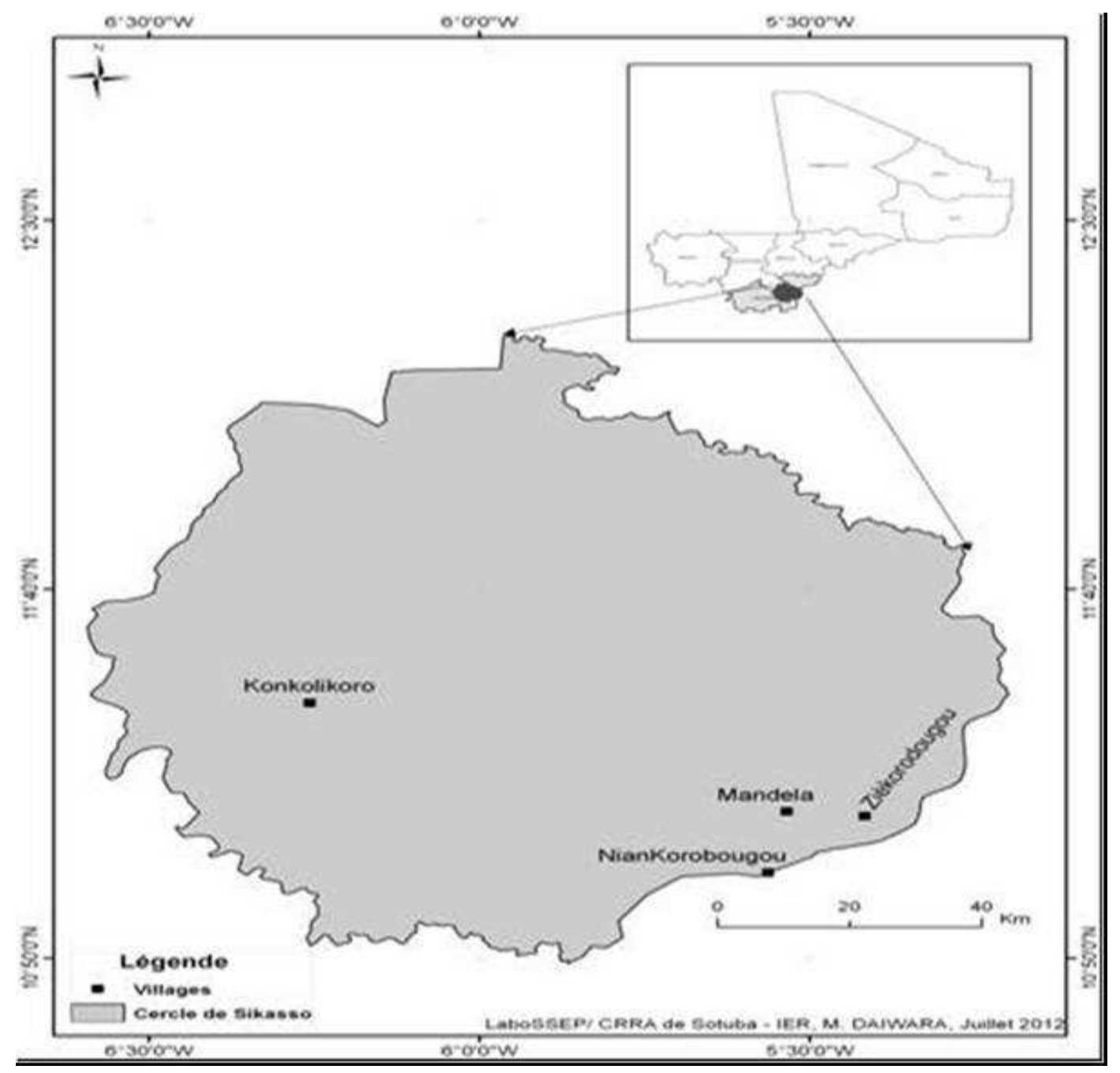

Figure 1 : Localisation de la zone d'étude dans le cercle de Sikasso au Sud du Mali.

Tableau 1 : Répartition des groupes ethniques par villages enquêtés.

\begin{tabular}{lcc}
\hline Villages & Groupes ethniques & Activités \\
\hline Konkolikoro & Bambara & Agriculture \\
& Peul & Agriculture \\
\hline Mandela & Peul & Elevage \\
& Samogo & Agriculture \\
\hline Niankorobougou & Bambara & Agriculture \\
& Peul & Agriculture \\
\hline Ziékorodougou & Peul & Agriculture \\
& Sénoufo & Agriculture \\
\hline
\end{tabular}


A. M. KOUYATE et al. / Int. J. Biol. Chem. Sci. 9(6): 2754-2763, 2015

Tableau 2 : Résultats de l'analyse de variance.

\begin{tabular}{|c|c|c|c|c|c|c|c|c|c|c|c|c|c|}
\hline Usages & $\begin{array}{l}\text { Paramètres } \\
\text { étudiés }\end{array}$ & $\begin{array}{l}\text { Carapa } \\
\text { procera }\end{array}$ & $\begin{array}{c}\text { Elaeis } \\
\text { guineensis }\end{array}$ & $\begin{array}{c}\text { Khaya } \\
\text { senegalensis }\end{array}$ & $\begin{array}{c}\text { Lophira } \\
\text { lanceolata }\end{array}$ & $\begin{array}{c}\text { Pentadesma } \\
\text { butyracea }\end{array}$ & $\begin{array}{l}\text { Vitellaria } \\
\text { paradoxa }\end{array}$ & $\begin{array}{c}\text { Blighia } \\
\text { sapida }\end{array}$ & $\begin{array}{c}\text { Borassus } \\
\text { aethiopum }\end{array}$ & $\begin{array}{c}\text { Lannea } \\
\text { microcarpa }\end{array}$ & $\begin{array}{c}\text { Raphia } \\
\text { sudanica }\end{array}$ & $\begin{array}{l}\text { Sterculia } \\
\text { setigera }\end{array}$ & $\begin{array}{c}\text { Ximenia } \\
\text { americana }\end{array}$ \\
\hline \multirow[t]{4}{*}{ Alimentaire } & Ethnie & 0,542 & $<0,000^{* *}$ & - & $0,006 * *$ & $0,004 * *$ & 0,642 & - & - & - & - & - & - \\
\hline & Villages & 0,469 & $0,012 *$ & - & $0,001 * *$ & $0,000 * *$ & 0,480 & - & - & - & - & - & - \\
\hline & Ages & 0,905 & 0,399 & - & $0,010^{*}$ & 0,113 & 0,717 & - & - & - & - & - & - \\
\hline & Sexes & 0,918 & 0,139 & - & 0,076 & $0,040^{*}$ & $0,043^{*}$ & - & - & - & - & - & - \\
\hline \multirow[t]{4}{*}{ Savon } & Ethnie & $0,012^{*}$ & $0,003 * *$ & $<0,000 * *$ & $0,020^{*}$ & 0,137 & 0,458 & - & - & - & - & - & - \\
\hline & Villages & $<0,000 * *$ & $0,005 * *$ & $<0,000 * *$ & $0,006^{* *}$ & $0,010^{*}$ & 0,613 & - & - & - & - & - & - \\
\hline & Ages & 0,113 & 0,832 & 0,458 & $0,005 * *$ & 0,458 & 0,800 & - & - & - & - & - & - \\
\hline & Sexes & 0,765 & 0,473 & $0,004 * *$ & 0,349 & 0,503 & 0,235 & - & - & - & - & - & - \\
\hline \multirow[t]{4}{*}{ Peau } & Ethnie & $0,021 *$ & 0,271 & - & $0,039^{*}$ & $0,003 * *$ & 0,177 & - & - & - & - & - & - \\
\hline & Villages & $0,032 *$ & 0,582 & - & 0,342 & 0,302 & $0,013^{*}$ & - & - & - & - & - & - \\
\hline & Ages & $<0,000 * *$ & 0,960 & - & 0,269 & 0,208 & 0,229 & - & - & - & - & - & - \\
\hline & Sexes & 0,481 & 0,183 & - & 0,075 & $0,029 *$ & $0,002 * *$ & - & - & - & - & - & - \\
\hline \multirow[t]{4}{*}{ Cheveux } & Ethnie & 0,218 & 0,081 & - & 0,255 & $0,006^{*}$ & 0,127 & - & - & - & - & - & - \\
\hline & Villages & 0,662 & 0,415 & - & $0,008^{*}$ & 0,131 & 0,245 & - & - & - & - & - & - \\
\hline & Ages & 0,173 & 0,509 & - & 0,352 & 0,131 & 0,115 & - & - & - & - & - & - \\
\hline & Sexes & 0,255 & 0,349 & - & $0,022 *$ & $0,029 *$ & $0,007 *$ & - & - & - & - & - & - \\
\hline \multirow[t]{4}{*}{ Médecine } & Ethnies & 0,058 & 0,260 & $0,017 *$ & 0,069 & 0,474 & 0,218 & 0,169 & - & - & - & - & - \\
\hline & Villages & $0,003 * *$ & 0,551 & 0,139 & 0,581 & 0,717 & 0,176 & 0,134 & - & - & - & - & - \\
\hline & Ages & $0,039 *$ & $0,004 * *$ & 0,764 & 0,201 & 0,369 & 0,261 & 0,434 & - & - & - & - & - \\
\hline & Sexes & 0,212 & 0,379 & 0,286 & 0,685 & 0,734 & $0,001 * *$ & 0,173 & - & - & - & - & - \\
\hline \multirow[t]{4}{*}{ Insecticide } & Ethnie & 0,084 & 0,786 & $<0,000^{* * *}$ & 0,095 & 0,490 & 0,055 & 0,126 & $0,026^{*}$ & $0,007 * *$ & $0,027 *$ & $0,003^{* *}$ & $0,014^{*}$ \\
\hline & Villages & 0,051 & 0,356 & $0,000 * *$ & 0,154 & 0,288 & $0,032^{*}$ & 0,153 & 0,576 & 0,066 & 0,179 & $0,014 *$ & 0,101 \\
\hline & Ages & 0,928 & 0,509 & 0,807 & 0,907 & 0,927 & 0,820 & 0,689 & 0,293 & 0,838 & 0,429 & 0,991 & 0,271 \\
\hline & Sexes & 0,101 & 0,349 & 0,086 & 0,285 & 0,228 & 0,169 & 0,321 & 0,441 & 0,252 & 0,194 & 0,089 & 0,355 \\
\hline
\end{tabular}




\section{DISCUSSION}

Les espèces d'arbres locaux à huile identifiées au Sud du Mali sont au nombre de 12 qui est inférieur à 16 fournis par Ouédraogo et al. (2013) à l'Ouest du Burkina Faso. Cependant, les deux études ont conclu à la forte citation de Vitellaria paradoxa, Carapa procera, Pentadesma butyracea et de Lophira lanceolata.

Les différences significatives observées entre les groupes ethniques pour les différentes utilisations dans le cadre de la présente étude ont été confirmées par Chadare et al. (2008) qui ont noté la variation des usages du baobab en fonction des groupes ethniques au Bénin.

L'âge et le sexe présentent un intérêt capital car ils impactent sur les stratégies locales de gestion des ressources naturelles. Leur prise en compte se justifie par le fait que l'exploitation des produits forestiers non ligneux est essentiellement une activité familiale, voire communautaire. Ceci explique les différences significatives observées en fonction de l'âge et du sexe dans le cadre de la présente étude. Ces résultats sont contraires à ceux obtenus au Bénin sur Adansonia digitata (De Caluwé et al., 2009).

En ce qui concerne les huiles alimentaires, la faible citation de Lophira lanceoalata par les jeunes et les adultes s'explique par le fait que cet usage est en pleine déperdition en milieu rural à cause de l'existence d'une gamme variée d'huiles modernes et raffinées. Cela confirme la forte citation de l'espèce par les vieilles personnes qui sont détentrices des pratiques culinaires ancestrales.

La forte citation de Vitellaria paradoxa par les hommes au détriment des femmes est due à l'intérêt économique du beurre. Ceci est un facteur incitatif amenant les hommes à devenir des acteurs importants dans la chaîne de valeurs.

La variation de la citation des espèces ligneuses alimentaires entre les villages s'expliquerait par l'abondance des espèces dans la zone d'étude et la diversité culturelle. La forte citation exclusive de Pentadesma butyracea à Ziékorodougou qui borde la galerie de Farako illustre bien cette variation. Olivier et al. (2012) ont indiqué que les usages alimentaires relèvent de la culture traditionnelle en pays Sénoufo.

En matière d'us et de coutumes au Mali, la cosmétique a été longtemps un domaine réservé aux femmes. C'est pourquoi, les espèces utilisées pour la fabrication du savon, les soins des cheveux et de la peau ont été grandement citées par les femmes. Des études réalisées dans la même zone d'étude ont confirmé les utilisations cosmétiques de Carapa procera surtout chez les femmes (Dembélé et al., 2015). Le partage de savoir traditionnel entre les femmes et les hommes a été également indiqué par la littérature (Kristensen et Lykke, 2003; Dembélé et al., 2015).

En plus de la forte utilisation de l'huile de Carapa procera pour les soins médicinaux qui a été confirmée par d'autres sources (Guèye et al., 2009; Weber et al., 2010; Dembélé et al., 2015), l'étude a révélé les vertus cosmétiques de l'huile de cette espèce (fabrication de savon, soins de la peau) qui sont loin d'être négligeables dans les villages enquêtés.

Le faible pourcentage de citation des espèces bio-insecticides peut s'expliquer par la forte utilisation des produits insecticides classiques dans les terroirs villageois.

La forte citation des espèces par les groupes ethniques pour les autres types d'usages serait due à leur similarité culturelle, car ces groupes partagent la même aire géographique. Ceci a été confirmé par Pfeiffer et Butz (2005) qui disent que l'origine géographique, entre autres, peut influencer la transmission des connaissances.

La présence de Vitellaria paradoxa dans tous les différents usages a été confirmée par d'autres auteurs (Diarassouba et al., 2008).

Le déficit de communication entre les jeunes, les adultes et les vieilles personnes pourrait expliquer la forte citation des espèces par les vieilles personnes pour tous les usages confondus. Cela peut constituer un gap dans la conservation durable des ressources génétiques forestières.

\section{Conclusion}

Les enquêtes ethnobotaniques quantitatives sur les arbres locaux à huile ont été menées au Sud du Mali. Les résultats ont 
montré une grande diversité d'espèces d'arbres utilisées à des fins alimentaires, cosmétiques, médicinales et insecticides. Les usages des huiles ont été influencés par les groupes ethniques, les villages, l'âge et le sexe. Les espèces d'arbres les plus citées par les communautés locales sont Carapa procera (savon, peau, médecine), Lophira lanceolata (alimentation, savon, peau, cheveux), Pentadesma butyracea (alimentation, savon, peau, cheveux) et Vitellaria paradoxa (alimentation, savon, peau, cheveux, médecine). Les savoirs traditionnels ainsi identifiés mettent en évidence les potentialités de ces espèces. Au regard de ce riche potentiel, les espèces susvisées doivent bénéficier des techniques d'aménagement et de gestion sylvicoles appropriées pour le bienêtre des communautés locales démunies.

\section{CONFLIT D'INTERET}

Les auteurs déclarent qu'il n'y a pas de conflit d'intérêt.

\section{CONTRIBUTIONS DES AUTEURS}

AMK a réalisé la conception du protocole, la recherche documentaire, la supervision des enquêtes, le traitement des données et la rédaction du manuscrit; UD a participé à la conception du protocole et la supervision des enquêtes. AML a participé à la conception du protocole, l'analyse des données et à la correction du manuscrit.

\section{REMERCIEMENTS}

Nos remerciements s'adressent aux habitants des villages d'études pour leur disponibilité et à toute l'équipe d'enquête.

\section{REFERENCES}

Abasse T, Weber JC, Katkore B, Boureima M, Larwanou M, Kalinganire A. 2011. Morphological variation in Balanites aegyptiaca fruits and seeds within and among parkland agroforests in eastern Niger. Agroforest. Syst., 81: 57-66. DOI : http://dx.doi.org/10.1007/s 10457-010-9323-x

Albert A, Emmanuel B. 2011. Improving nutrition and health through non-timber forest products in Ghana. J. Health. Popul. Nutr., 29: 141-148. DOI: http://dx.doi.org/10.3329/jhpn.v29i2.78 56

Andel TV. 2006. Les Produits Forestiers autres que le Bois d'Euvre. La Valeur des Plantes Sauvages. Agromisa Publication and CTA, Wageningen, Pays Bas.

Bikoue MAC, Essomba H. 2007. Gestion des ressources naturelles fournissant les produits forestiers non ligneux alimentaires en Afrique centrale. Rome, Italie.

Biloso MA, Lejoly J. 2006.Etude de l'exploitation et du marché des produits forestiers non ligneux à Kinshasa. Tropicultura, 24(3): 183-188.

Chadare FJ, Hounhouigan JD, Linnerman AR, Nout MJR, van Boekel MAJS. 2008. Indigenous Knowledge and Processing of Adansonia Digitata L. Food Products in Benin. Ecol. Food Nutr., 47(4): 338-362. DOI: http://dx.doi. org/10.1080/03670240802003850

Dagnelie P. 1977. Analyse statistique à plusieurs variables. Presses agronomiques de Gembloux. Belgique.

Dawson IK, Lengkeek K, Weber JC, Jamnadass R. 2009. Managing genetic variation in tropical trees, linking knowledge with action in agroforestry ecosystems for improved conservation and enhanced livelihoods. Biodivers. Conserv., 18(4): 969-986. DOI: http://dx.doi.org/10.1007/s10531008-9516-z

De Caluwé E, De Smedt S, Assogbadjo AE, Samson R, Sinsin B, Van Damme P. 2009. Ethnic differences in use value and use patterns of baobab (Adansonia digitata L.) in northern Benin. Afr. J. Ecol., 47(3): 433-440. DOI: http://dx.doi.org/10.1111/j.13652028.2008.01023.x

Dembélé U, Lykke AM, Koné Y, Témé B, Kouyaté AM. 2015. Use-value and importance of socio-cultural knowledge on Carapa procera trees in the sudanian zone in Mali. J. Ethnobiol. Ethnomed., 11-14. DOI : http://dx.doi.org/10.1186/1746-426911-14 
Diarassouba N, Koffi KE, N'Guessan KA, Van Damme P, Sangare A. 2008. Connaissances locales et leur utilisation dans la gestion des parcs à karité en Côte d'Ivoire. Afrika Focus, 21: 77-96.

Dossou ME, Houessou GL, Lougbégnon OT, Tenté AHB, Codja JTC. 2012Etude ethnobotanique des ressources forestières ligneuses de la forêt marécageuse d'Agonvè et terroirs connexes au Bénin. Tropicultura, 30(1): 41-48.

Dovie DBK. 2003. Rural economy and livelihoods from the non-timber forest products trade, Compromising sustainability in southern Africa. Int. J. Sust. Dev. World Ecol., 10(3): 247-262. DOI: http://dx.doi.org/10.1080/ 13504500309469803

Dronne Y, Forslund A. 2009. Le rôle croissant des huiles tropicales sur les marchés internationaux, Principaux acteurs et produits. OCL., 16(4): 184-192. DOI : http://dx.doi.org/10.1051/ocl.2009.026 7.

DRPSIAP. 2014. Rapport annuel. Direction Régionale de la Planification, de la Statistique et de l'informatique, de l'Aménagement du Territoire et de la Population de Sikasso. Mali.

FAO. 2007. Situation des Forêts du Monde 2007. FAO : Rome, Italie.

FAO. 2009. Forests and Poverty Reduction. FAO : Rome, Italie.

Gaoué OG, Ticktin T. 2009. Fulani knowledge of the ecological impacts of Khaya senegalensis (Meliaceae) foliage harvest in Benin and its implications for sustainable harvest. Econ. Bot., 63(3): 256-270.

Gruère G, Giuliani A, Smale M. 2006. Marketing underutilized plant species for the benefit of the poor, Washington, USA.

Guèye M, Kenfac D, Forget PM. 2009. Importance socioculturelle, potentialités économiques et thérapeutiques du Carapa (Meliaceae) au Sénégal. In Systematics and Conservation of African Plants. Royal Botanic Garden, Kew; 359-367.
Johnson RA, Wichern DW. 1998. Applied Multivariate Statistical Analysis. Prentice-Hall, Inc.

Kristensen M, Lykke AM. 2003. Informantbased valuation of use and conservation preferences of savanna trees in Burkina Faso. Econ. Bot., 57: 203-217. DOI: 10.1663/0013-0001(2003)057 [0203:IVOUAC]2.0.CO;2

Krohmer J, Hahn-Hadjali K, Wittig R. 2006. Utilisation de la flore sauvage par des populations peules au Burkina Faso et au Bénin. Etudes Flor. Vég. Burkina Faso., 10: 33-48.

Lykke AM, Kristensen MK, Ganaba S. 2004. Valuation of local use and dynamics of 56 woody species in the Sahel. Biodivers. Conserv., 13:19611990. DOI: 10.1023/B:BIOC. $0000035876.39587 .1 \mathrm{a}$

Mahapatra AK, Albers AK, Robinson EJZ. 2005. The Impact of NTFP Sales on Rural Households' Cash Income in India's Dry Deciduous Forest. Environ. Manage., $\quad 35(3)$ : 258-265. DOI: http://dx.doi.org/10.1007/s00267003-8203-9

Medeiros MFT, Sandos DA SILVA P, Albuquerque UP, 2011. Quantification in ethno-botanical research. An overview of indices used from 19952009. Sintientibus ser. Ci. Biol., 11(2): 211-230. DOI:10.13102/scb108

Olivier M, Zerbo P, Boussim JI, Guinko S. 2012. Les plantes des galeries forestières à usage traditionnel par les tradipraticiens de santé et les chasseurs Dozo Sénoufo du Burkina Faso. Int. J. Biol. Chem. Sci., 6(5): 2170-2191. DOI : http://dx.doi.org/10.4314/ijbcs.v6i5.24

Ouédroago A, Lykke AM, Lankoandé B, Korbéogo G. 2013. Potentials for promoting oil products identified from traditional knowledge of native trees in Burkina Faso. Ethnobot Res Appl., 11: 071-083. www.ethnobotanyjournal. org/vol11/i1547-3465-11-071.pdf

Pfeiffer JM, Butz RJ. 2005. Assessing cultural and ecological variation in Ethnobiological Research. The Importance of Gender. J. Ethnobiol., 25(2): 240-278. DOI : http://dx.doi. 
org/10.2993/0278-0771(2005)25[240-

ACAEVI]2.0.CO;2

Philippeau G. 1986. Comment Interpréter les Résultats d'une Analyse en composantes Principales? ITCF. Service des études statistiques. STATITCF.

PROMISAM. 2007. Synthèse des plans communaux de sécurité alimentaire du cercle de Sikasso 2007-2011. PROMISAM, Mali.

Schumann K, Wittig R, Thiombiano A, Becker U, Hahn K. 2011. Impact of land-use type and harvesting on population structure of a non-timber forest product-providing tree in a semiarid savanna, West Africa. Biol. Conserv., 144(9): 2369-2376. DOI: http://dx.doi.org/10.1016/j.biocon .2011 .06 .018
Tchatat M, Ndoye O. 2006. Etude des produits forestiers non ligneux d'Afrique Centrale: réalités et perspectives. Bois For. Trop., 288(2): 27-39.

Weber N, Birnbaum P, Forget PM, Gueye M, Kenfacks D. 2010. L'huile de Carapa (Carapa spp. Meliaceae) en Afrique de l'Ouest. Utilisations et implications dans la conservation des peuplements naturels. Fruits., 65(6): 343-354. DOI : http://dx.doi.org/10.1051/fruits/201002 9

Wong JLG, Thornber K, Baker N. 2001. Evaluation des ressources en produits forestiers non ligneux. Produits forestiers non ligneux 13. Expérience et principes de biométrie: Rome. 